\title{
Operative Management of Ventriculoperitoneal Shunts During Bariatric Operations
}

Sigrid Bairdain ${ }^{1}$, Richard S. Flint ${ }^{2}$, Alexandra J. Golby ${ }^{3}$, Suzanne A. Tharin ${ }^{3}$, David B. Lautz 4

1. Department of Surgery, Boston Children's Hospital 2. Department of Surgery, Brigham \& Women's Hospital 3. Department of Neurosurgery and Radiology, Brigham \& Women's Hospital, Harvard Medical School 4. Department of Surgery, Emerson-Mass General Hospital, Harvard Medical School

$\square$ Corresponding author: Sigrid Bairdain, sbairda@yahoo.com

Disclosures can be found in Additional Information at the end of the article

\section{Abstract}

Background: Idiopathic intracranial hypertension is a condition associated with morbid obesity. Although bariatric surgery offers an effective treatment, some bariatric surgical candidates will present with indwelling ventriculoperitoneal (VP) shunts. The maintenance of shunt sterility is paramount.

Objective: To review our experience with a regimen of VP shunt externalization, in patients undergoing primary bariatric procedures.

Setting: Academic university hospital

Methods: Records of all patients who underwent bariatric procedures between 4/1/2004 and $11 / 1 / 2007$ were reviewed. Our protocol was to externalize all shunts at the beginning, for patients undergoing Roux-en-Y gastric bypasses (RYGB), and to leave all shunts externalized for one week; shunts were re-internalized at a subsequent procedure if no signs of infection were present. Laparoscopic adjustable gastric banding (LAGB) patients would have the shunt evaluated simultaneously and left in place if functional.

Results: Of the 1,759 patients who underwent bariatric procedures from 2004 to 2007, six had indwelling VP shunt. Four patients underwent laparoscopic RYGB, 1an open RYGB, and one patient underwent LAGB with the same VP shunt management protocol. All patients had their shunts re-internalized without sequelae, with the exception of one patient who was no longer shunt dependent. One patient did have migration outside of the abdominal wall by the shunt; to prevent migration, the shunt was fixed to a section of prolene mesh. No patients developed evidence of contamination.

Conclusions: The technique of VP shunt externalization may be a safe and effective technique of managing VP shunt during bariatric surgical procedures. 
Idiopathic intracranial hypertension (IIH), or pseudotumor cerebri, is a clinical condition shown to be associated with obesity and the female gender [1-2]. Idiopathic intracranial hypertension is defined as a clinical syndrome of raised intracranial pressure in the absence of a space-occupying lesion, without enlargement of the cerebral ventricles for which no causative factor can be identified [3]. As the prevalence of obesity increases, it is likely that clinicians will encounter more of this previously uncommon condition. Traditionally, IIH is most commonly treated with medication within the carbonic anhydrase inhibitor family [3]. However, surgical management has been shown to be useful in resolving the symptoms of IIH, especially in patients with morbid obesity [4].

Ventriculoperitoneal (VP) shunts have become increasingly popular in the diversion of cerebrospinal fluid for treating IIH. The presence of a VP shunt could pose an increased risk of shunt-related complications in patients undergoing complex intra-abdominal surgical procedures like Roux-en-Y gastric bypass (RYGB). As a result of manipulation of the VP shunt during a weight loss operation, the risk of infection either intra-operatively or from postoperative anastomotic leaks may lead to devastating complications, such as meningitis, shunt blockage, and uncontrolled rise in intracranial pressure. In addition, VP shunts have been shown to have a higher rate of failure in morbidly obese patients, which further complicates the management of these patients [5].

A potential solution to this dilemma is to temporarily externalize the shunt as has been reported in other surgical procedures [6-7]. The VP shunt is reinserted at a later date, after the period of highest contamination risk. This technique has the added advantage of enabling the evaluation of shunt function and the potential for shunt independence. This paper describes our experience in managing VP shunts during bariatric surgical procedures.

\section{Materials And Methods}

Under our institution's review board approved protocol, all patients undergoing RYGB or laparoscopic adjustable gastric banding (LAGB) between April 1, 2004 and November 1, 2007 were identified from the prospective, longitudinal institutional database. Those that also had a VP shunt at the same time were selected. Brigham and Women's Hospital protocol was \#P000704; Boston Childrens Hospital protocol was \#P00007528. All patients met the criteria for bariatric surgery established by the National Institutes of Health Consensus Development Panel [8]. All had failed to maintain weight loss by non-surgical means. The patient decided upon the type of operation (RYGB, laparoscopic RYGB, or LAGB) after attending a public information session and personal consultation with the surgeon.

At the beginning of the surgical procedure in the operating room, the distal portion of the VP shunt was externalized from the right upper quadrant incision site or clavicle incisions site (depending on the judgment of the surgeon) and the passage of clear cerebrospinal fluid (CSF) was confirmed. Then, it was connected to an external ventricular drainage system that was maintained for the duration of shunt externalization. The weight-loss operation was then conducted per our routine. The VP shunt was clamped and symptoms of elevated intracranial pressure (ICP) were queried for a week following surgery. If the patient was asymptomatic following 48-hours of clamping the VP shunt, then the VP shunt was removed. If, however, the patient was symptomatic, then the VP shunt was re-inserted into the abdomen by standard general and neurosurgical techniques. Antibiotics were prescribed during the duration of the externalization; however, cerebrospinal fluid was not routinely sent in the operating room.

All laparoscopic RYGB (LRYGB) patients had the creation of a small gastric pouch (approximately $20 \mathrm{cc}$ in volume, as judged by the surgeon), with a $100 \mathrm{~cm}$ antecolic Roux limb, and $50 \mathrm{~cm}$ afferent limb. All LAGB patients had the Lap-Band system (ALLERGAN, Santa Barbara, CA) placed by experienced surgeons using the pars flaccida technique [9]. 
Postoperative recovery was in a bariatric surgical ward with neurosurgical consultation. Patients were discharged on a dietician-led nutrition plan and reviewed in outpatient clinic at regular intervals. Gastric banding adjustments were made in the office per standard protocol. All data were prospectively entered into a computerized database and reviewed retrospectively.

Queries were made for patient demographics, age, gender, initial body mass index (BMI), perioperative mortality (defined as death by any cause within 30 days of operation), and perioperative complications (anastomotic leak, deep venous thrombosis, pulmonary embolism, gastrointestinal tract bleeding requiring transfusion or endoscopy, wound infection requiring antibiotics, need for splenectomy, incisional hernia, bowel obstruction, anastomotic stricture, gastric prolapse, and band erosion).

\section{Results}

Of the 1,759 patients that underwent primary bariatric surgical procedures during the study period, six had indwelling shunts (five were for IIH and one was for post-subarachnoid hemorrhage hydrocephalus). Five of the six patients were female, while one was male. Mean age of this small cohort was $39+/-10$ years of age. All patients had VP shunt externalization at the time of their surgery. Four of six patients underwent LRYGB, one underwent an open RYGB, and one underwent LAGB. There were no recorded mortalities or complications.

There were no VP shunt malfunctions related to infectious complications. However, one patient required multiple attempts of VP shunt re-insertion. This patient had a long history of multiple VP shunt revisions for recurrent displacements from the peritoneal space. At the time of her LRYGB, her shunt was externalized via an incision at the clavicle, but was difficult to retract as the distal intra-abdominal end had been secured to a mesh at a prior revision. Therefore, $10 \mathrm{~cm}$ of catheter was withdrawn and the shunt was amputated at this point. Further management for the patient included re-internalization of her VP shunt through clavicular and RUQ incisions on postoperative day nine. The patient subsequently required two revisions of the distal end of VP shunt due to displacement from the peritoneal space, associated with subcutaneous CSF collection. There have been no more shunt complications in this patient.

In addition, in the process of externalization, VP shunts may be ligated or removed in newly shunt-independent patients. One patient (LRYGB with VP shunt for subarachnoid hemorrhage hydrocephalus) tolerated clamping of the VP shunt. We were able to ligate the shunt and chose not to remove the entire shunt system at that time to minimize the number of incisions and the risk of further complications.

\section{Discussion}

Bariatric surgery has become increasingly prevalent as clinicians recognize its ability to induce significant and sustained weight loss with the amelioration of obesity-related co-morbidities [10-11]. With the increasing prevalence of morbid obesity and its association with IIH, the frequency that bariatric surgeons encounter IIH is concurrently increasing [4]. In this study, we describe our experience with temporarily externalizing VP shunt at the time of LRYGB, RYGB, or LAGB. In our small series of six patients, we have demonstrated this as a potentially safe and effective technique of managing VP shunts during weight-loss operations.

The estimated incidence of IIH is 21.4 per 100,000 obese females with BMI >30kg/m2 [12]. Overall, the incidence of IIH in overweight patients has been shown to increase with increasing BMI [12-13]. It has been hypothesized that obese patients are at an increased risk because of a number of reasons, including a proposed association with cerebral edema as a result of disturbances in neuroendocrine physiology leading to increased brain water content [14-15]. 
There may also be an association between extra-ovarian production of estrone in obese patients and risk of IIH [16]. Increased intra-abdominal pressures may increase the risk of IIH in morbidly obese patients [17]. This may lead to elevation of the diaphragm with an increase in intra-thoracic pressure, leading to a reduction in cerebral venous drainage to the heart via the jugular venous system [17-18].

Worsening obesity is associated with poorer visual outcomes, especially in individuals with BMI $>40 \mathrm{~kg} / \mathrm{m}^{2}$ [1]. Again, symptoms may be distressing and permanent neurological damage can occur, such as trigeminal neuralgia, Bell's palsy, and the dreaded sequelae of permanent visual loss. In addition, morbidly obese patients are more likely to have a persistence of other IIH symptoms despite treatment as compared to non-obese patients [19]. Diagnosis relies on the modified Dandy criteria (increased intracranial pressure with opening pressure $>20 \mathrm{~cm} \mathrm{H}_{2} \mathrm{O}$, clinical signs of raised ICP, normal CSF composition, and normal radiological cranial imaging)[20].

Given the retrospective nature of our study, we only evaluated those patients with surgical interventions. However, treatment options for IIH include symptomatic relief with analgesics or efforts to reduce ICP that range from medications, such as acetazolamide, to surgical interventions, such as optic nerve sheath fenestration or shunting (VP or lumboperitoneal) [21]. Treatment options have also included transverse sinus stenting with complete resolution of symptoms in select patients [22]. Outcome studies following bariatric surgery have also been shown to yield complete resolution of IIH symptoms [23]. Idiopathic intracranial hypertension, as an indication for adult shunt surgery, increased from $1.8 \%$ of all adult shunt surgeries in the U.S. in 1998 to $4.5 \%$ in 2002 [6], paralleling the increasing prevalence of morbid obesity. In the same time period, there was an increase in the number of bariatric surgical procedures performed in patients with IIH [6]. With the increasing prevalence of obesity, the number of patients undergoing bariatric surgical procedures for IIH who have VP shunts will also be expected to increase.

The resolution of IIH in morbidly obese patients following weight-loss operations has led to an increased demand for bariatric surgery to become standard of care in morbidly obese patients with IIH [23], especially since it has been shown that these patients have a persistence of IIH symptoms following other forms of treatment [19, 24]. Morbidly obese patients with preexisting VP shunt for IIH presenting for a weight-loss operation represent a therapeutic dilemma. The decision to externalize or not to externalize the VP shunt varies across institutions and arises largely because of feared complications, which include an increase in ICP from shunt valve malfunction/failure and/or infection, which may occur due to a potentially contaminated surgical field [25].

A potential risk exists for increased intra-abdominal pressure created by the carbon dioxide pneumoperitoneum to cause increased back pressure in patients with VP shunt, which may be transferred to the ventricular cavities leading to increased ICP. With severe increase in back pressure, there is also a theoretical risk of shunt valve rupture. Some VP shunt valves can withstand pressures up to $300 \mathrm{mmHg}$, and the intra-abdominal pressures created because of the carbon dioxide pneumoperitoneum $(15-18 \mathrm{mmHg}$ ) will be unlikely to cause a rupture [26]. Neale, et al. showed that this complication was highly unlikely. In their study, VP shunt valves that ruptured did so at pressures exceeding $80 \mathrm{mmHg}$, far greater than intra-abdominal pressures typically created during laparoscopy [27].

Jackman, et al., in their series of 18 patients with pre-existing VP shunt who had laparoscopic surgery, showed that there was no clinical evidence of increased ICP; however, in their study, no patient underwent invasive intracranial pressure monitoring. Heart rate and blood pressure changes were used as proxies for increases in ICP [25]. Brown and colleagues acknowledge that 
laparoscopy appears to be safe in patients with VP shunt undergoing procedures that are less than three hours in length [7]. Some authors have recommended that patients be placed in a steep Trendelenburg position to facilitate CSF drainage intra-operatively. Brown and his group recommended preoperative neurosurgical evaluation to determine the appropriateness of VP shunt externalization or steep Trendelenburg in this unique group of patients. However, weight-loss operations vary in duration, and for more complex and longer procedures like revisions, the risk of increased ICP definitely should not be overlooked. The average duration of surgery in our series was within the threshold duration recommended by Brown and his colleagues to potentially externalize the VP shunt. For example, the median operative time in this small cohort was 4:20 minutes (range: 2 hours - 5:26 minutes).

Finally, there is risk of contamination of the surgical field and dissemination of diseasecausing microorganisms to the central nervous system. In our small series of six patients, we showed that externalization of the VP shunt during the procedure is a potentially reasonably safe option serving two major purposes: removing the shunt from the potentially contaminated surgical site and facilitation of shunt function assessment and dependence. This technique has been safely utilized in other surgical procedures [7]. In fact, one series suggested that for routine clean and clean-contaminated cases, that there is minimal risk for VP shunt malfunction or infection; thus, there is no reason for shunt externalization [28]. In this particular series, there were only two infections out of 26 cases [28]; however, the definition of VP shunt in adults is variable and the quoted overall incidence ranges from approximately $2 \%$ to $17 \%[29]$.

We found it to be a simple technique requiring no special maneuvers. Our only issue was that of "shunt pull-out" in a single patient while trying to reinsert the shunt. Shunt pull-outs, or displacements from the peritoneal space, are probably a common complication in morbidly obese patients, likely due to pannus movement and repeated traction on the catheter. In this patient, a Prolene mesh was ultimately placed at the distal end of the patient's shunt to keep it successfully in place; similar to Morrison, et al. description of reducing complications related to distal shunt placement associated with obesity [30]. In addition, VP shunt may be ligated or removed in newly shunt-independent patients. We were able to ligate the shunt in one of our patients who had been shunted for post-subarachnoid hydrocephalus, but no longer required the shunt. We chose not to remove the entire shunt system at that time to minimize the number of incisions and risk of complication.

\section{Conclusions}

In conclusion, we described our experience with temporarily externalizing VP shunt at the time of RYGB or LAGB procedures. We demonstrated that this is a safe and effective technique of managing VP shunt during bariatric surgical procedures in our small series of six patients. We acknowledge that there may be other factors that are paramount, including the overall time of the procedure or the effect of contamination and/or translocation; therefore, the ultimate decision may be resigned to the operating surgeon regarding the risk:benefit ratio of this temporizing procedure. Further studies are needed to address VP shunt management after surgery and whether there are statistically significant differences in EBWL, which leads to changes in their management.

\section{Additional Information \\ Disclosures}

Human subjects: Consent was obtained by all participants in this study. Brigham and Women's Hospital issued approval Brigham and Women's Hospital protocol \#P000704; Boston Childrens Hospital protocol \#P00007528. Under our institution's review board approved protocol, all 
patients undergoing RYGB or laparoscopic adjustable gastric banding (LAGB) between 4/1/2004 and 11/1/2007 were identified from the prospective, longitudinal institutional database. Those that also had a VP shunt at the same time were selected. All patients met the criteria for bariatric surgery established by the National Institutes of Health Consensus Development Panel. Animal subjects: All authors have confirmed that this study did not involve animal subjects or tissue. Conflicts of interest: In compliance with the ICMJE uniform disclosure form, all authors declare the following: Payment/services info: All authors have declared that no financial support was received from any organization for the submitted work. Financial relationships: All authors have declared that they have no financial relationships at present or within the previous three years with any organizations that might have an interest in the submitted work. Other relationships: All authors have declared that there are no other relationships or activities that could appear to have influenced the submitted work.

\section{References}

1. Rowe FJ, Sarkies NJ: The relationship between obesity and idiopathic intracranial hypertension. Int J Obes Relat Metab Disord. 1999, 223:54-9.

2. Ireland B, Corbett JJ, Wallace RB: The search for causes of idiopathic intracranial hypertension. A preliminary case-control study. Arch Neurol. 1990, 47:315-20.

3. Ball AK, Clarke CE: Idiopathic intracranial hypertension. Lancet Neurol. 2006, 5:433-42.

4. Sugerman HJ, Felton WL 3rd, Sismanis A, Kellum JM, DeMaria EJ, Sugerman EL: Gastric surgery for pseudotumor cerebri associated with severe obesity. Ann Surg. 1999, 229:634-40.

5. Burgett RA, Purvin VA, Kawasaki A: Lumboperitoneal shunting for pseudotumor cerebri. Neurology. 1997, 49:734-9.

6. Curry WT Jr, Butler WE, Barker FG 2nd: Rapidly rising incidence of cerebrospinal fluid shunting procedures for idiopathic intracranial hypertension in the United States, 1988-2002. Neurosurg. 2005, 57:97-108.

7. Brown JA, Medlock MD, Dahl DM: Ventriculoperitoneal shunt externalization during laparoscopic prostatectomy. Urology. 2004, 63:1183-5.

8. No authors listed: NIH conference. Gastrointestinal surgery for severe obesity. Consensus Development Conference Panel. Ann Intern Med. 1991, 115:956-61.

9. SAGES Webmaster Library Images 2010. The Pars Flaccida Technique . (2010). Accessed: August 26, 2012: http://www.sages.org/image-library/the-pars-flaccida-technique/.

10. Buchwald H, Avidor Y, Braunwald E, Jensen MD, Pories W, Fahrbach K, Schoelles K: Bariatric surgery: A systematic review and meta-analysis. JAMA. 2004, 292:1724-37.

11. Santry HP, Gillen DL, Lauderdale DS: Trends in bariatric surgical procedures. JAMA. 2005, 294:1909-17.

12. Radhakrishnan K, Thacker AK, Bohlaga NH, Maloo JC, Gerryo SE: Epidemiology of idiopathic intracranial hypertension: A prospective and case-control study. J Neurol Sci. 1993, 116:18-28.

13. Durcan FJ, Corbett JJ, Wall M: The incidence of pseudotumor cerebri. Population studies in Iowa and Louisiana. Arch Neurol. 1988, 45:875-7.

14. Raichle ME, Grubb RL Jr, Phelps ME, Gado MH, Caronna JJ: Cerebral hemodynamics and metabolism in pseudotumor cerebri. Ann Neurol. 1978, 4:104-11.

15. Reid AC, Matheson MS, Teasdale G: Volume of the ventricles in benign intracranial hypertension. Lancet. 1980, 2:7-8.

16. Newton M, Cooper BT: Benign intracranial hypertension during prednisolone treatment for inflammatory bowel disease. Gut. 1994, 35:423-5.

17. Bloomfield GL, Ridings PC, Blocher CR, Marmarou A, Sugerman HJ: A proposed relationship between increased intra-abdominal, intrathoracic, and intracranial pressure. Crit Care Med. 1997, 25:496-503.

18. Bloomfield GL, Dalton JM, Sugerman HJ, Ridings PC, DeMaria EJ, Bullock R: Treatment of increasing intracranial pressure secondary to the acute abdominal compartment syndrome in a patient with combined abdominal and head trauma. J Trauma. 1995, 39:1168-70.

19. Reid AC, Thomson JA: Absence of significant endocrine deficiencies in benign intracranial hypertension. J Neurol Neurosurg Psychiatry. 1981, 44:731-4.

20. Shin RK, Balcer LJ: Idiopathic Intracranial Hypertension. Curr Treat Options Neurol. 2002, 4:297-305. 
21. Skau M, Brennum J, Gjerris F, Jensen R: What is new about idiopathic intracranial hypertension? An updated review of mechanism and treatment. Cephalalgia. 2006, 26:384-99.

22. Ahmed RM, Wilkinson M, Parker GD, Thurtell MJ, Macdonald J, McCluskey PJ, Allan R, Dunne V, Hanlon M, Owler BK, Halmagyi GM: Transverse sinus stenting for idiopathic intracranial hypertension: a review of 52 patients and of model predictions. AJNR Am J Neuroradiol. 2011, 32:1408-14. 10.3174/ajnr.A2575

23. Sugerman HJ, Felton WL 3rd, Salvant JB Jr, Sismanis A, Kellum JM: Effects of surgically induced weight loss on idiopathic intracranial hypertension in morbid obesity. Neurology. 1995, 45:1655-9.

24. Wall M, George D: Idiopathic intracranial hypertension. A prospective study of 50 patients . Brain. 1991, 114:155-80.

25. Jackman SV, Weingart JD, Kinsman SL, Docimo SG: Laparoscopic surgery in patients with ventriculoperitoneal shunts: Safety and monitoring. J Urol. 2000, 164:1352-4.

26. Collure DW, Bumpers HL, Luchette FA, Weaver WL, Hoover EL: Laparoscopic cholecystectomy in patients with ventriculoperitoneal (VP) shunts. Surg Endosc. 1995, 9:409-10.

27. Neale ML, Falk GL: In vitro assessment of back pressure on ventriculoperitoneal shunt valves. Is laparoscopy safe?. Surg Endosc. 1999, 13:512-5.

28. Li G, Dutta S: Perioperative management of ventriculoperitoneal shunts during abdominal surgery. Surg Neurol. 2008, 70:492-5. 10.1016/j.surneu.2007.08.050

29. Wells DL, Allen JM: Ventriculoperitoneal shunt infections in adult patients. AACN Adv Crit Care. 2013, 24:6-12. 10.1097/NCI.0b013e31827be1d1

30. Morrison JF, Sung KE, Bergman AM, Rosenblatt MS, Arle JE: A novel solution to reduce the complications of distal shunt catheter displacement associated with obesity. J Neurosurg. 2010, 113:1314-6. 10.3171/2010.6.JNS10300 